\title{
construcción de túneles mediante máquinas que excavan a sección total (tuneladora) y normas para su utilización
}

G. Vié, Ingeniero de Minas

$579-20$

sinopsis

En este articulo se analizan algunos resultados interesantes obtenidos por el empleo de máquinas de perforación continua en varias obras de reciente ejecución, así como diversas mejoras realizadas después de diferentes estudios y ensayos.

De este análisis se deduce que estas máquinas sólo son rentables para grandes longitudes y que es preciso realizar estudios geológicos previos antes de comenzar la perforación.

Entre las meioras producidas están: la utilización de muelas activadas, con las que se consigue sustituir las fuerzas de presión estática por procesos dinámicos; la colocación de chorros de agua a alta presión junto a las herramientas de perforación con lo que se aumenta la potencia de corte; el uso del transporte hidráulico en galerías de pequeño diámetro; el empleo de resina aminoplasta armada con fibras de vidrio para aumentar la estanquidad y mejorar el revestimiento de los túneles; etc.

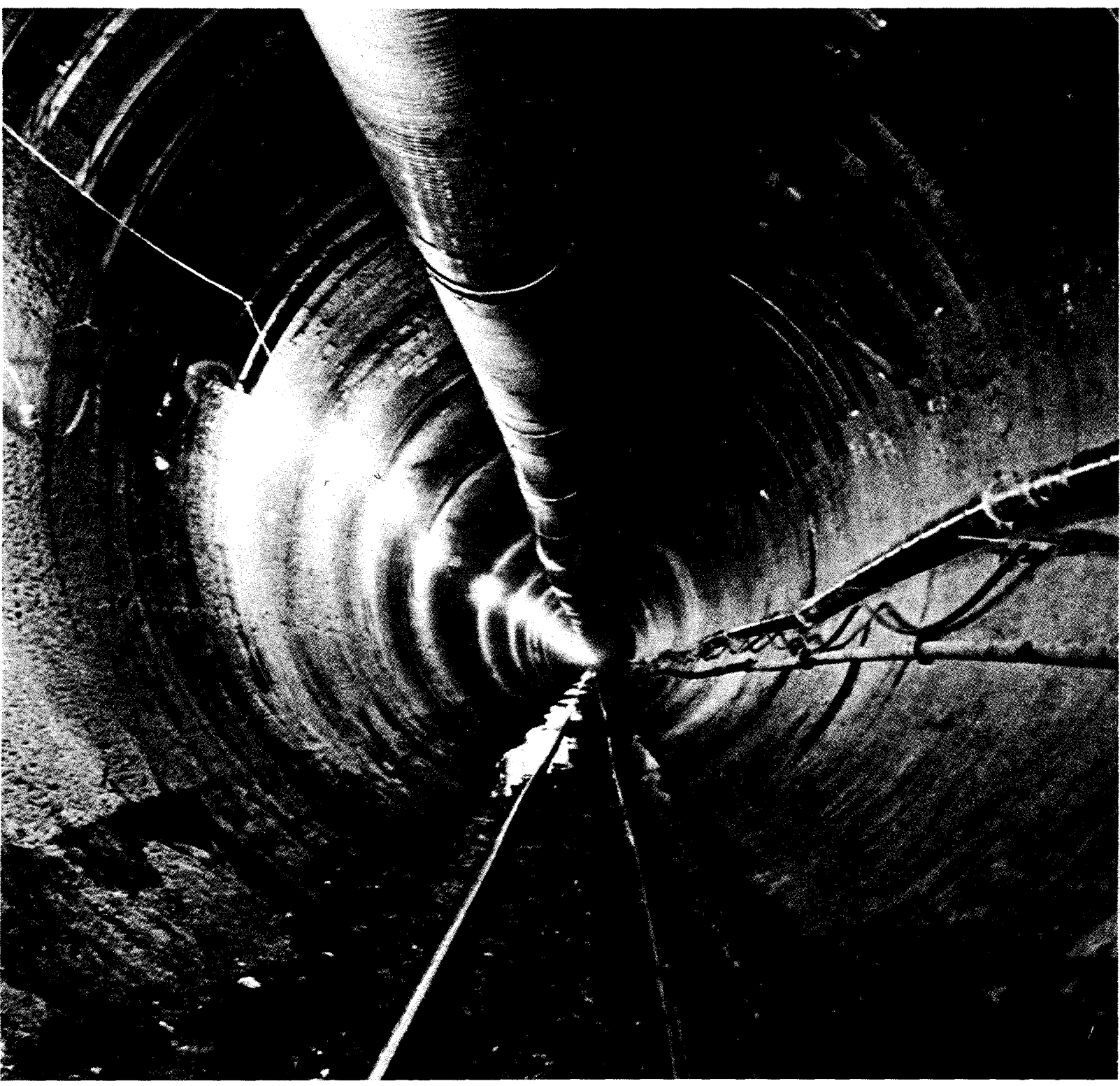

Galeria, de $9.740 \mathrm{~m}$ de longitud y $5,60 \mathrm{~m}$ de diametro, excavada mediante maquina tuneladora en una zona de esquistos y de gres situada bajo el lago Huron, en la ciudad de Detroit. Velocidad media horaria de avance: 4,18 metros. 
En el $n .^{\circ} 314$ de Informes de la Construcción, de octubre de 1979 , se efectuó una descripción de las máquinas tipo "minador continuon, indicando algunos resultados interesantes obtenidos por ellas en varias obras de reciente ejecución.

Es conveniente completar ese trabajo con la relación de nuevas obras, con algunas mejoras conseguidas después de diversos estudios y ensayos, entre los que merecen una atención especial los que tratan de la destrucción de las rocas por muelas de corte.

Desde luego, el empleo de una máquina de este tipo sólo es rentable en una excavación de gran longitud pues, aunque no es posible amortizar su precio de compra, si se puede en gran manera contribuir a ello.

Los gastos de montaje y, a menudo, los de puesta a punto para el comienzo de los trabajos, aunque están incluidos en las cláusulas de ajuste de precio alzado de los gastos de instalación de la obra, casi siempre resultan insuficientes debido a los imprevistos.

Los equipos son pesados, las potencias puestas en obra necesitan transportes largos y de un coste elevado, etc...

El conocimiento de las condiciones geológicas locales es esencial, pues la aptitud de una roca para dejarse excavar es función de sus propiedades macroestructurales.

Es muy importante poder ir definiendo durante el avance de los trabajos un módulo de elasticidad, un límite de rotura a tracción y la superficie sometida a compresión.

Está claro que un corte de muela, bien concebido, hará posible alcanzar el valor más elevado del esfuerzo de compresión compatible con la resistencia, así como una mayor duración de los bordes de esta herramienta.

Determinada la resistencia a compresión de la roca, es posible fijar el valor medio de la carga por muela y, por tanto, calcular sin riesgo de error la concepción de aquélla y las tensiones impuestas a sus rodamientos.

La separación entre muelas está ligada al limite de rotura a tracción de la roca considerada y, según sea esta separación, así resultarán las mayores dimensiones de los fragmentos obtenidos. Igualmente hay que tener en cuenta la esquistosidad y las posibles fisuraciones de esta misma roca. Consideradas la carga media y la separación entre muelas se puede fijar el número de aquéllas sobre el disco de corte.

El empuje de la máquina será función de la relación entre la carga y la duración de los rodamientos de las ruedas así como del número de aquéllas.

La potencia de una máquina tuneladora se define por la velocidad de avance de la máquina y por la de desgaste de las herramientas. La velocidad diaria de avance es el producto de la velocidad de penetración ( $\mathrm{m} /$ hora máquina) por la tasa de utilización (horas de trabajo de la máquina por turno). Desde luego, las caracteristicas de la máquina se deben elegir en función de la naturaleza de la roca y las condiciones de trabajo.

En los años próximos serán cada vez mayores las longitudes de las obras subterráneas por lo que se deberá prestar una gran atención al precio de coste por metro perforado, el cual viene dado, en una proporción muy elevada, por las operaciones de corte y desintegración de la roca cuando su resistencia sobrepasa un cierto valor, por ejemplo, $20.000 \mathrm{MPa}$ en terreno no abrasivo y $15.000 \mathrm{MPa}$ en formación abrasiva.

Las máquinas tuneladoras con disco de muelas son interesantes debido a su aptitud para perforar a sección total en los niveles geológicos con resistencia superior a esos límites, por la rapidez del avance que se obtiene y por la supresión de algunos peligros que se derivan de la perforación diaria.

En la travesia de los terrenos más duros, el número de muelas no se puede aumentar más de lo que permita la superficie disponible en el disco de ataque, lo que hace que las excavaciones de pequeña sección no sean precisamente las más fáciles.

Es importante tener en cuenta este postulado de base. Bajo el esfuerzo de presión y en la línea horizontal, formada por el par de rotación del disco de corte, cada muela quebranta un trozo de roca situado junto a la zona de contacto y provoca su rotura por cizallamiento. 


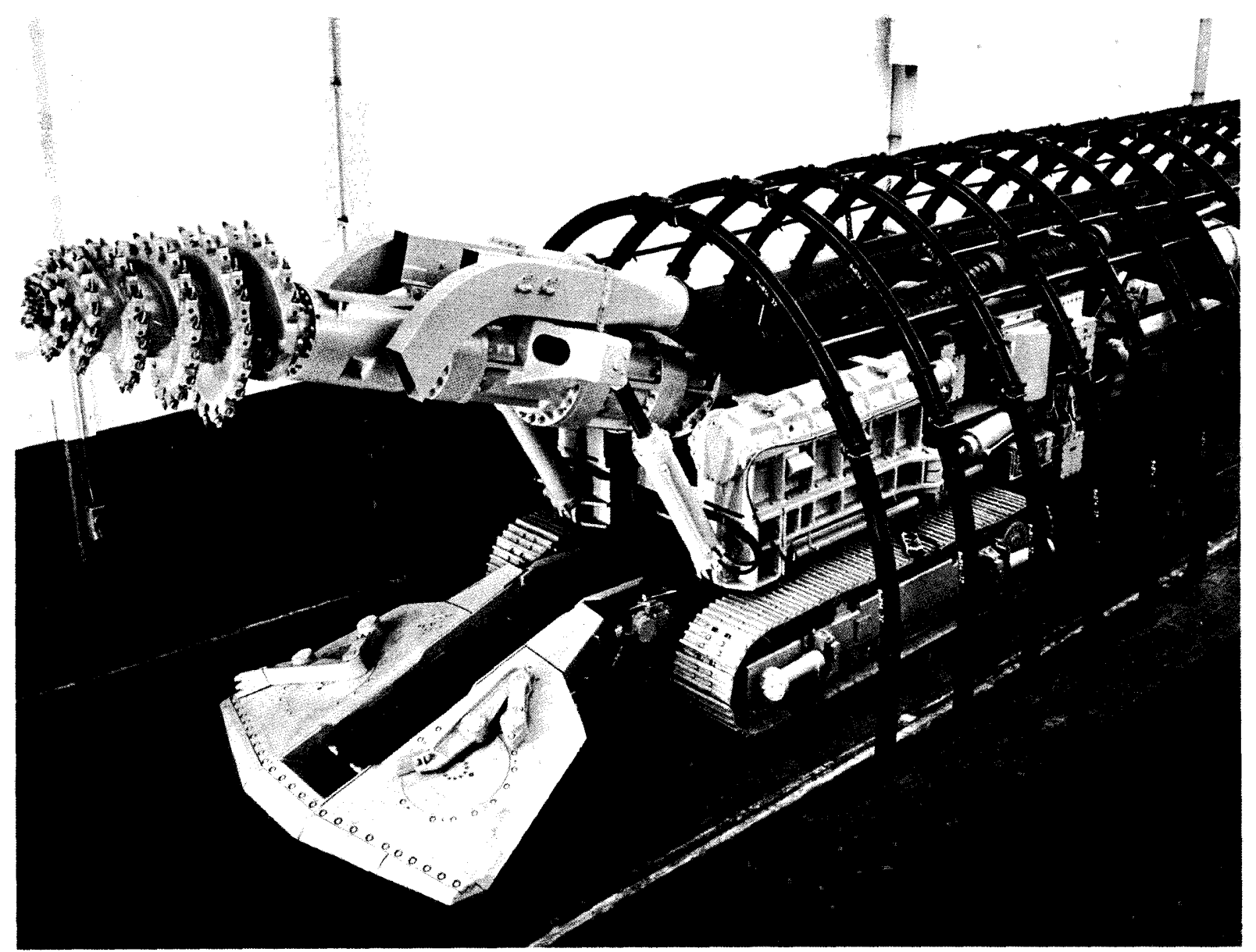

Máquina EICKHOFF, tipo EV-100-B, empleada para excavación continua de galerías y túneles. Esta vista muestra cómo una máquina de este tipo puede ser empleada en galerias de pequeña sección.

Una muela que pasa varias veces por un mismo surco, bajo una carga cortante, en cada una de las pasadas sucesivas destruye menos masa rocosa que en las anteriores.

Existe una separación óptima entre dos surcos próximos, para la cual el grosor de los fragmentos es máximo y la energía consumida por volumen de roca abatida se reduce de una manera importante.

A pesar de la amplitud de los ensayos realizados con objeto de definir los mejores parámetros, la manera de conseguir la disposición más eficaz, asi como el tamaño idóneo de las muelas, es mediante aproximaciones sucesivas durante la travesía de una roca dada.

Las informaciones geológicas indispensables para un buen conocimiento de las rocas se obtienen por medio de perforaciones de extracción de testigos y, a ser posible, con coronas diamantadas.

Las muestras se deben estudiar cuidadosamente para conseguir el máximo de ense- ñanzas, las cuales serán los elementos determinantes en la elección de muelas, su colocación, su número, etc...

Una mejora conseguida gracias a las recientes experimentaciones es la de las muelas activadas, término por el cual debe entenderse la activación de una herramienta mediante la incorporación de un elemento giratorio que produzca un desequilibrio dinámico, sustituyendo, de esta forma, las fuerzas de presión estática por procesos dinámicos.

Se obtienen asi fuerzas centrífugas que permiten triturar mejor las rocas más duras, como pueden ser, un granito muy siliceo y no alterado; dioritas cuarciferas y cuarcitas libres.

Las dimensiones de las muelas activadas pueden llegar a ser invariables si se utiliza un sistema excitador accionado neumática o hidráulicamente. 
Estas muelas activadas se deben fijar en número suficiente sobre el platillo de corte. Para obtener fuerzas centrifugas más importantes pueden hacerse de mayores dimensiones.

Desde hace algún tiempo se han realizado experimentos con objeto de estudiar la posibilidad de asociar el trabajo de las muelas de corte con el de chorros de agua a alta presión para disminuir la presión en el frente y la potencia de la máquina tuneladora y conseguir, de esta manera, máquinas menos pesadas, menos voluminosas y más manejables.

Un chorro de agua a alta presión tiene una potencia elevada de corte que resulta de la transmisión de energía de una superficie de contacto puntual entre el chorro de agua y el perímetro de excavación en la roca maciza.

Con estos chorros de agua a presión se producen, a ambos lados de la muela de corte, desprendimientos, al contacto de los cuales la muela puede hacer cizallar las aristas de roca situadas entre dichas entallas.

Observando la incidencia de diferentes factores: presión de agua, diámetro y disposición de los conductos de inyección del agua en el frente, a la altura del platillo de corte, se obtiene una reducción del 30 al $50 \%$ de la presión y de la potencia.

A esta técnica nueva se le llama hidromecánica y es muy eficaz en roca dura.

Desde luego, la máquina tuneladora debe poder funcionar sin chorros, o sea, que si se desconecta la parte hidráulica, la máquina no por eso deja de excavar.

La profundidad de corte obtenido utilizando chorros de agua a alta presión es función de la presión, del número y de la velocidad relativa de los chorros, así como de la dureza de la roca.

Esta profundidad de corte es mucho mayor cuando el chorro se coloca detrás, o incluso sobre la muela, que si se sitúa entre los discos.

Los discos pulverizan la roca que queda asi más receptiva a la acción del agua, con lo que se puede aumentar la eficacia hasta en un $50 \%$.
A una profundidad dada se puede reducir la presión necesaria cuando la roca ha sido, por así decirlo, pretriturada por la muela. En efecto, para una profundidad de $3 \mathrm{~mm}$, es preciso un chorro de $21.000 \mathrm{MPa}$ sobre una superficie sin previa pretrituración mientras que sólo se necesita un chorro de $7.000 \mathrm{MPa}$ si se ha efectuado ésta.

Ejemplo: Una máquina WIRTH con 2,60 m de diámetro y equipada con un platillo de corte que lleva diversas muelas acopladas en dos discos necesita cien conductos de agua a presión reunidos en varios grupos. La producción de agua a presión es de 120 litros/min, con una presión de 35.000 ó 40.000 MPa conseguidos mediante la ayuda de cuatro bombas de impulsión, con una potencia instalada de un millar de $\mathrm{kW}$. Los conductos de inyección tienen un diámetro de 0,25 a $0,63 \mathrm{~mm}$.

De una manera general se debe prever, para la instalación de bombas de agua a alta presión, una potencia de 150 a $200 \mathrm{~kW}$ por metro cuadrado de sección.

Teniendo en cuenta esta exigencia, es evidente que, para grandes diámetros de excavación, no se necesitan demasiadas instalaciones, sobre todo a la vista de las ventajas que se consiguen. La experiencia muestra las posibilidades económicas de la transformación.

Una muela de la periferia de la cabeza de corte, si gira a razón de $10 \mathrm{ciclos} / \mathrm{min}$, puede estar sometida a una velocidad de $2 \mathrm{~m} / \mathrm{seg}$.

La profundidad de corte exclusivamente mecánico en roca dura puede ser inferior a $3 \mathrm{~mm}$ en cada revolución.

Como el desgaste de las muelas o discos cortantes es mayor en el centro y en la periferia, es lógico colocar los chorros en esas zonas con objeto de reducir las fuerzas de presión de desgaste.

Según se ha demostrado experimentalmente, la mejor disposición de los chorros es entre las muelas de corte.

Una máquina con un diámetro de excavación de $6 \mathrm{~m}$ y la cabeza o platillo de corte equipado con 50 muelas admitirá, por ejemplo, 75 chorros con $28.000 \mathrm{MPa}$ y una potencia total del orden de un millar de C.V. 
En Africa del Sur se excavó, mediante una máquina WIRTH, una galería de $1.150 \mathrm{~m}$ de longitud y $3,30 \mathrm{~m}$ de diámetro en un terreno de cuarcita dura y abrasiva. Su rendimiento alcanzó $18,7 \mathrm{~m}$ en jornadas de tres turnos de 8 horas y hasta $7 \mathrm{~m}$ por turno.

La máquina, que medía $76 \mathrm{~m}$ de longitud, estaba equipada con una cabeza de perforación de 12 t de peso, la cual llevaba 23 herramientas de carburo. La presión total ejercida sobre el frente de avance era de 600 toneladas.

Queda, de esta manera, comprobado que un minador continuo puede perforar sin dificultad en roca muy dura, pero esta demostración resultó costosa puesto que el precio de ejecución fue seis veces superior al de una excavación realizada por el método clásico de explosivos.

Esto último confirma la hipótesis de que sólo debe utilizarse el perforador continuo en grandes longitudes, en las cuales se puede incluso obtener cierta economía.

La firma alemana DEMAG proporcionó para su utilización, en una explotación minera de Rumania, una máquina tuneladora TVM$45 \mathrm{H}$ de $46 \mathrm{~m}$ de longitud y $250 \mathrm{t}$ de peso, y con un diámetro de excavación de 4,80 metros.

El platillo de ataque podía estar equipado con muelas dobles o simples, que ejercen una presión de 9,2 t cada una.

Se comprobó que las muelas de corte situadas en la periferia del platillo debian reemplazarse muy frecuentemente.

Ese platillo, que podia tener 4,20 ó $5,50 \mathrm{~m}$ de diámetro, giraba a una velocidad de 6,3 ciclos/min, con un par de $800 \mathrm{KN} \cdot \mathrm{m}$.

En esta explotación, la máquina fue rentable pues se trataba de una galería de 16 kilómetros de longitud.

Esta misma firma Mannesmann - Demag construyó, para una explotación carbonífera, una máquina con el objeto de excavar una galería de $17 \mathrm{~km}$ de longitud y $6 \mathrm{me}-$ tros de diámetro, que atraviesa esquistos y conglomerados.

La cabeza de perforación gira a razón de $4,9 \mathrm{ciclos} / \mathrm{min}$, velocidad conseguida mediante cuatro motores de $160 \mathrm{~kW}$ cada uno.
Alrededor de una pieza central se colocaron muelas en uno o dos discos, según la naturaleza del terreno. La máquina tiene una longitud de $36 \mathrm{~m}$ y $275 \mathrm{t}$ de peso. La sección es suficiente para evacuar los escombros con la ayuda de una cinta transportadora instalada en la parte baja. La presión en los circuitos hidráulicos de la máquina es de $2.000 \mathrm{MPa}$.

Para las minas Hans Aden (RFA), la misma firma alemana entregó, a finales de febrero de 1980 , una máquina de $6,50 \mathrm{~m}$ de diámetro para la excavación de 19,2 km de galería en roca a unos $1.000 \mathrm{~m}$ de profundidad. En estos terrenos el sostenimiento por medio de cimbras circulares se sustituye por un bulonado inmediato que asegura la seguridad del personal alrededor de la máquina.

Desde luego, el empleo de una máquina de este tipo en terreno dificil trae consigo muchos problemas.

Podemos citar, por ejemplo, el paso de fallas con bolsas de arcilla que pueden alcanzar muchos metros de longitud, donde los patines de anclaje de la máquina se deslizan sobre los paneles, a lo que hay que unir las dificultades de evacuación de los bloques de arcilla y las medidas indispensables de sostenimiento.

Un problema idéntico es el de la travesía de una zona de rocas fuertemente trituradas, comprendida entre dos fallas, o una red de pequeñas bolsas de escombros o el avance a través de gres fisurado.

Es, pues, importante la realización de estudios previos del perfil longitudinal del túnel.

Puede llegar a ocurrir que en un terreno difícil sea interesante prever un escudo con techo corto que permita la colocación de marcos y cercos metálicos.

Existe un proyecto israeli de excavación, mediante máquina tuneladora, de una galería de $14 \mathrm{~km}$ de longitud y $4,50 \mathrm{~m}$ de diámetro con revestimiento hormigonado o 5 metros de diámetro sin ningún tipo de revestimiento.

Este trabajo seria para unir el Mar Muerto $y$ el Mar Mediterráneo con un caudal de agua de $30 \mathrm{~m}^{3} / \mathrm{seg}$. 
La galería pasaría bajo los Montes de Judea y desembocaría en el Mar Muerto a la cota $-80 \mathrm{~m}$, mientras que el nivel actual se encuentra por debajo de $-400 \mathrm{~m}$.

El estudio geológico preliminar indica que el $60 \%$ de las excavaciones se efectuaría en rocas débiles y el otro $40 \%$ en rocas duras.

La compañia ELECTRICIDAD DE FRANCIA decidió, durante los años anteriores, mejorar el actual salto de agua de POUGET (Departamento de Avegnon), en el nordeste de Saint-Affrique, creando algunas nuevas obras de aprovechamiento hidroeléctrico:

- Una toma de agua en el embalse de VILLEFRANCHE DE PANAT, en la ALRANCE.
- Una galería inclinada con una pendiente de 0,6 por mil para asegurar la unión entre dicho embalse y el de SaintAmans, y una galería que enlaza el embalse de Saint-Amans con una nueva conducción forzada.

Se eligió como máquina excavadora el perforador continuo WIRTH a sección total, del tipo TBIV $475-505 \mathrm{H}$.

La sección circular de la galería es de 5,05 metros en las zonas no revestidas y de $4,70 \mathrm{~m}$ en las zonas revestidas; su longitud es del orden de $4.750 \mathrm{~m}$. Está situada a la derecha de una galeria anteriormente excavada y a una distancia entre 40 y 100 metros.

Las condiciones geológicas locales conforman un terreno muy dificil. Si se hubiese seguido el avance clásico por explosivos se habrian ocasionado unos sobreperfiles exagerados.

Las características de esta máquina WIRTH son las siguientes:

- Potencia de mando

- Régimen (regulable de manera continua)

- Par de rotación

- Carrera de perforación.

- Fuerza de perforación

- Número de patines de apriete sobre las paredes ... 8

- Presión hidráulica de apriete.

- Potencia eléctrica total instalada

- Potencia de los transformadores...
$3 \times 200 \mathrm{~kW}$

0 a 1 vueltas/minuto

$60.000 \mathrm{~m} . \mathrm{kg}$

$1.250 \mathrm{~mm}$

Hasta $490 \mathrm{t}$

8

$225 \mathrm{kp} / \mathrm{cm}^{2}$

$650 \mathrm{~kW}$

$2 \times 500 \mathrm{kVA}$
La cabeza de perforación lleva un sistema de palas con cangilones. Las muelas centrales se agrupan en una placa central bulonada sobre la cabeza.

Tres agujeros de hombre, de $585 \mathrm{~mm}$ de diámetro, permiten el acceso al frente de corte. Seis cangilones situados en el entorno de la cabeza evacuan los escombros.

Esta máquina alemana fue comprada por EDF en 1978, a un precio de 200 millones de pesetas. Se puso en obra el mes de junio de 1980 en dos puestos de trabajo.

Durante la segunda quincena de junio de 1980 el avance fue de 50 metros.

En julio la excavación avanzó $69 \mathrm{~m}$ y en agosto de 1980, $128 \mathrm{~m}$. En septiembre de
1980 el avance conseguido fue de 147 metros. El 15 de octubre se alcanzaba el punto 825 , desde el principio del ataque.

Los resultados son, desde luego, satisfactorios.

Mannesmann - Demag tiene una gama completa de máquinas tuneladoras para excavar a sección total con seis diámetros estándar que varian desde $\varnothing 2,4 \mathrm{~m}$ a $\varnothing 2,7 \mathrm{~m}$.

Para secciones de excavación más importantes la construcción de máquinas es igualmente concebible.

Recientemente, tres perforadoras a sección total en servicio en las minas de carbón de la RFA excavaron $15 \mathrm{~km}$ de galerias de $6 \mathrm{~m}$ de diámetro. 
Estas máquinas llevan un sostenimiento anular de acero que permite a los mineros trabajar con toda seguridad inmediatamente detrás de la cabeza de perforación.

En algunos casos es difícil resolver el problema de evacuación de escombros, sobre todo en galerias de sección circular de pequeño diámetro realizadas en roca.

Los transportadores normales en esas secciones reducidas son de difícil empleo.

La solución quizá podria estar en el transporte hidráulico. Esta solución fue la aplicada en la excavación de una galería de $2,30 \mathrm{~m}$ de diámetro y $4.000 \mathrm{~m}$ de longitud, con una pendiente del $5 \%$.

Los escombros salidos de la máquina se recogian en un transportador repartidor $y$ se vertian en cajones donde se efectuaba su mezcla con agua.

Después de la eliminación sobre rastrillo de los fragmentos que excedían de los $60 \mathrm{~mm}$, la mezcla agua-escombros se impelía a una presión de $100 \mathrm{MPa}$ por dos bombas en serie en un conducto que desembocaba en el exterior.

La máquina de construcción alemana EICKHOFF, tipo EV-100 B es la adecuada, debido a su relativa simplicidad para la travesía de galerías de minas y de túneles de sección casi rectangular, por ejemplo, de $5,4 \mathrm{~m}$ de altura, 5,6 $\mathrm{m}$ de cabeza, 6,20 $\mathrm{m}$ en los paramentos ligeramente curvados de forma de herradura y $5,20 \mathrm{~m}$ en bóveda, o sea, alrededor de $30 \mathrm{~m}^{2}$ de sección. Pero puede también excavar cuando se trata de secciones semicirculares de gálibo más restringido $\left(14 \mathrm{~m}^{2}\right)$.

La particularidad de su brazo de extracción es que puede trabajar en roca muy dura, sin vibración perceptible y sin crear sobreperfiles.

Montada sobre orugas, su gran movilidad da incluso la posibilidad de excavar secciones que pasan los $30 \mathrm{~m}^{2}$.
El brazo de extracción está provisto de un tambor cónico con una profundidad de corte de $1,5 \mathrm{~m}$.

Los útiles que recubren ese tambor son de metal duro, embutidos en unas carcasas capaces de sentir los esfuerzos mayores derivados del ataque a la roca.

El tambor se acciona mediante un motor con número de placas variables y de una potencia comprendida entre 60 y $120 \mathrm{~kW}$.

El reductor con cuatro trenes de engranaje reduce las velocidades a 12,5 y $25 \mathrm{ci}$ clos/min, que equivalen a velocidades de corte de 0,7 y $1,4 \mathrm{~m} / \mathrm{seg}$. medidos en el diámetro medio del tambor.

Esta máquina pesa $65 \mathrm{t}$ con una potencia total instalada de $300 \mathrm{~kW}$. Esta potencia incluye la necesaria para el dispositivo de reamasado y de evacuación de los escombros por medio de una cinta transportadora unida al bastidor.

El túnel de carretera de Frejus, abierto a la circulación en julio de 1980 fue, al menos en parte, excavado por un perforador continuo tipo EICKHOFF, con dos brazos paralelos y simétricos.

Las galerías excavadas con máquinas tuneladoras tienen generalmente un buen comportamiento frente a la presión de los terrenos.

Pero algunas veces es necesario protegerlas mediante un revestimiento, sobre todo para asegurar su estanquidad. El agua es el elemento más perjudicial en trabajos subterráneos, especialmente cuando las excavaciones se realizan por el método convencional de explosivos.

En la travesia de terrenos más o menos acuíferos, la estanquidad puede ser indispensable, como ocurre en el túnel de carretera de Seehisberg (Alpes Suizos, entre Bâle y Milán), formado por dos galerías unidireccionales de $9.250 \mathrm{~m}$ de longitud.

La impermeabilidad se consiguió proyectando con pistola una emulsión betún - látex y, en algunas zonas, empleando láminas impermeables flexibles y delgadas rea- 


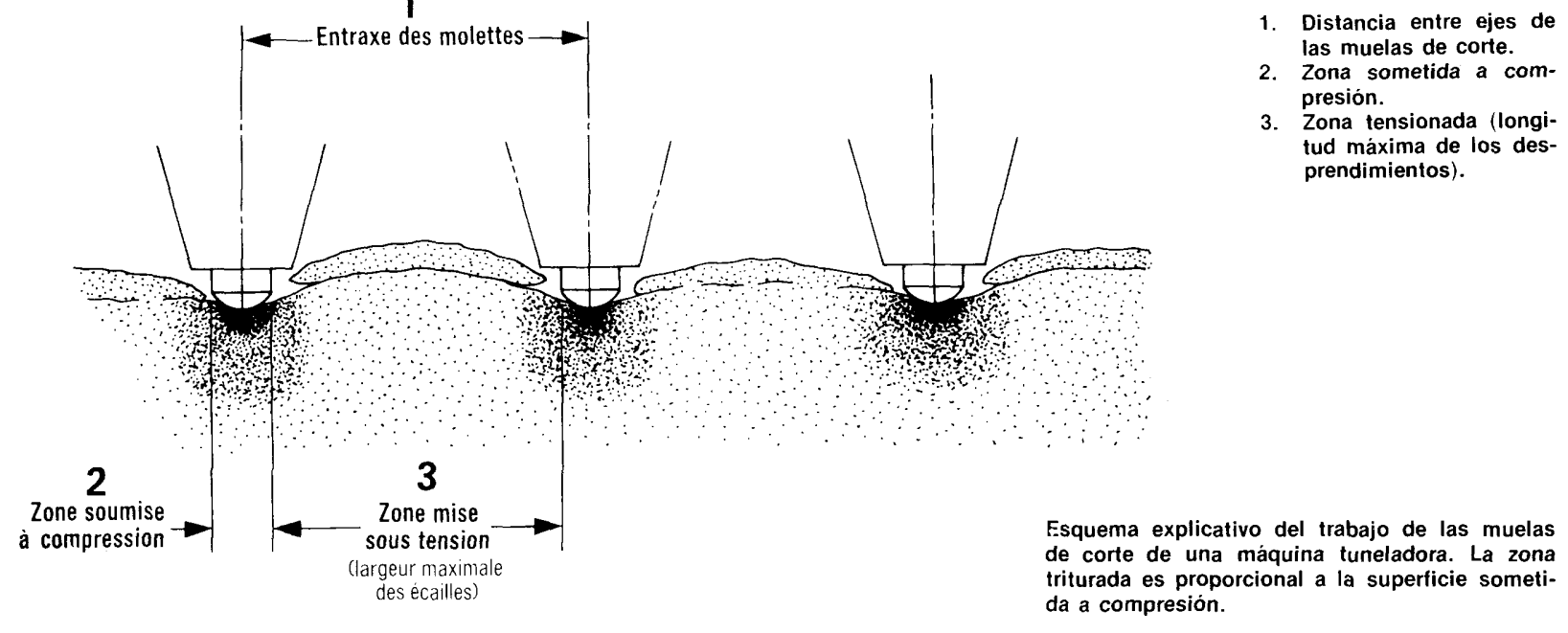

lizadas con elastómero Hypalon de la firma americana DU PONT.

La proyección del hormigón, hasta un espesor del orden de $3 \mathrm{~cm}$, es una técnica cada vez más habitual.

Lo que se pretende es mejorar la resistencia mecánica de ese sostenimiento mezclando fibras de acero en el hormigón proyectado.

Sin embargo, este método no ha tenido el desarrollo esperado, pues un cierto número de problemas prácticos de puesta en servicio dificultan su aplicación.

Por eso parece preferible la pulverización de resina sintética armada de fibras de vidrio ampliamente experimentada en las minas de hierro de Lorraine.

Fue necesario investigar una resina no tóxica en razón del trabajo en atmósfera confinada.

Esta fue la razón por la que se eliminaron las resinas epoxi y poliéster, tóxicas en medio cerrado, y que exigian además, al menos para la limpieza de los aparatos de proyección, el empleo de disolventes peligrosos para el personal.

Además existía el inconveniente de que son resinas muy caras cuando uno de los objetivos previstos es la reducción del precio de coste.

Las resinas anteriormente citadas son muy difíciles de bombear debido a que necesitan adición de cargas minerales en una cierta proporción.
Finalmente se eligió una resina aminoplasta, bajo la forma de un polvo blanco, no tóxico e ininflamable que se mezcla con el agua para su puesta en obra.

Después de la pulverización, las fibras de vidrio de una longitud media de $25 \mathrm{~mm}$ se proyectan sobre la resina. Se cortan en trozos de $25 \mathrm{~mm}$ por un cortador colocado sobre la pistola.

Para la pulverización se utiliza una bomba de doble efecto, con un caudal de $250 \mathrm{li}$ tros/hora y $5 \mathrm{~m}^{3} /$ minuto de consumo de aire a $50 \mathrm{MPa}$ de presión.

Con sólo dos obreros se consiguen rendimientos de $30 \mathrm{~m}^{2}$ de revestimiento por hora en dos pasadas sucesivas que dan $3 \mathrm{~mm}$ de espesor.

La resistencia a compresión de un revestimiento realizado así es de $590 \mathrm{kp} / \mathrm{m}^{2}$ y a tracción de $465 \mathrm{kp} / \mathrm{m}^{2}$.

Esta resistencia se logra de un modo más rápido que la del hormigón proyectado, pues se obtienen resistencias a compresión del orden de los $100 \mathrm{MPa}$ con sólo una hora de endurecimiento contra $500 \mathrm{MPa}$ en el mismo tiempo para el hormigón proyectado.

En las minas de hierro del Este de Francia, el precio por metro cuadrado es un $60 \%$ del obtenido con el hormigón proyectado clásico.

La proyección de resina aminoplasta armada de fibras de vidrio no protege solamente de la humedad, sino que incluso sustituye al entramado metálico asociado al bulona- 
do, utilizado para luchar contra el desconchado de los terrenos.

Si ese procedimiento no puede asegurar un sostenimiento portante en el sentido técnico del término, sí produce a veces una estanquidad satisfactoria que se opone a la degradación de los terrenos. nueva pulverización en la bóveda antes del hormigonado final.

Se ha estudiado la eventual toxicidad de las fibras de vidrio como la de todas las fibras minerales.

Mientras que algunas de estas fibras pue-
Esquema de la activación de una herra. mienta equipada con muelas de corte. Se consigue la sustitución de las fuerzas de presión estática por procesos dinámicos.

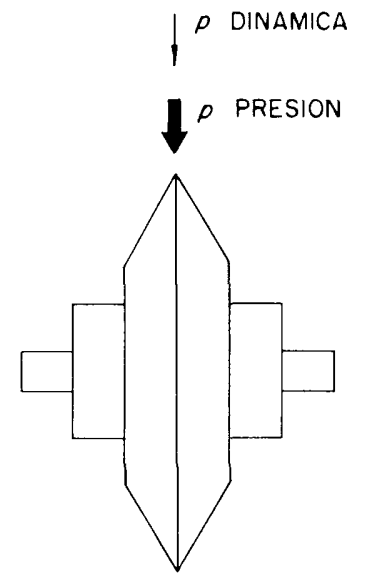

MUELA DE CORTE CLASICA

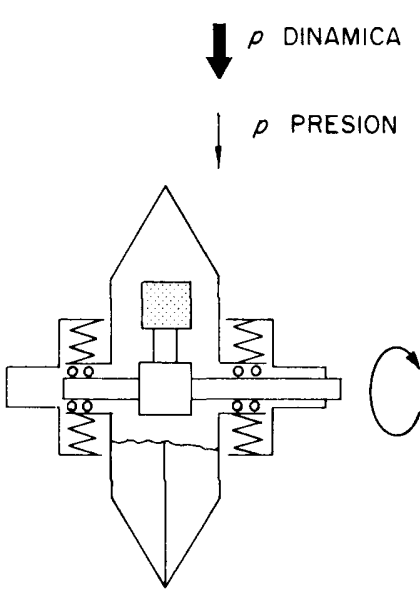

MUELA DE CORTE ACTIVADA
En el túnel de Frejus (Costa Itálica) un pozo de ventilación de $700 \mathrm{~m}$ de desarrollo se excavó mediante máquinas tunelacioras con una sección base de $3 \mathrm{~m}$ de diámetro. En la bóveda de este túnel se empleó una pulverización de resina armada de fibras de vidrio para evitar el desconchado $y$, posteriormente, se realizó un bulonado parcial.

La excavación continuó hasta la sección definitiva de $5,80 \mathrm{~m}$ de diámetro, con una den ser causa de afecciones cancerosas, las fibras de vidrio sólo provocan bronquitis pulmonares o irritaciones epidémicas.

Las fibras que se utilizan para su inyección en galería son de unos grosores bastante importantes, por lo que no contienen elementos respirables.

Una precaución útil, desde luego, es usar máscara.

\section{résumé}

CONSTRUCTION DES TUNNELS PAR MACHINES PLEINE SECTION (TUNNELERS) ET NORMES DE LEUR UTILISATION

G. Vié, ingénieur des mines

\begin{abstract}
dans cet article, l'auteur fait une analyse de quelques résultats intéressants obtenus par l'utilisation de mineurs continus sur divers chantiers récents, ainsi que de quelques perfectionnements apportés à la suite perfectionnements

De cette analyse il est déduit que ces machines ne sont rentables que pour de grandes longueurs et qu'il faut effecteur des études géologiques avant de procéder au forage.

Parmi les perfectionnements apportés figurent l'utilisation de molettes activées permettant le remplacement des forces de pression statique par des processus dynamiques, la disposition de jets d'eau à haute pression avec les outils de forage, ce qui permet d'augmenter la puissance de decoupage, l'utilisation du transport hydraulique en galeries de petit diamètre, l'emploi de résine aminoplaste armée de fibres de verre pour augmenter l'étanchéité et améliorer le revêtement des tunnels, etc.
\end{abstract}

\section{summary}

TUNNEL BUILDING WITH MACHINES THAT EXCAVATE A COMPLETE SECTION (TUNNELING MA CHINE) AND STANDARDS FOR USE

\section{G. Vié, Mining Engineer}

In this article an analysis is made of severa interesting results obtained by the use of nonstop drilling machines in various recent works, as well as different improvements carried out following a number od studies and tests.

From this analysis it can be concluded that these machines are only profitable for extensive lengths, and that it is necessary to carry out prior geological studies before commencing drilling operations.

Among the improvements are found: the use of activated teeth, whereby the static pressure forces are replaced by dynamic processes; the placement of high-pressure water blasts next to the drilling tools, thus increasing the cutting power; the use of hydraulic transport in small-diameter galleries; the use of aminoplast resin reinforced with fiberglass in order to enhance leakproofness and to improve the lining of the tunnels; etc.

\section{zusammenfassung}

TUNNELBAU MIT HILFE VON GESAMTQUERS CHITTBAGGERN (TUNNELMASCHINEN) UND NORMEN FUER DIE ANWENDUNG

G. Vié, Bergbauingenieur

In diesem Artikel werden einige interessante Ergebnisse analysiert, die auf Grund der Anwendung von Maschinen durchgehender Bohrung bei verschiedenen Bauarbeiten letzter Zeit erhalten wurden sowie verschiedene Verbesserungen, die nach mehreren Proben und Studien angewandt wurden.

Aus dieser Analyse koennen wir folgern, dass diese Maschinen nur fuer lange Strecken rentabel sind und es unbedingt erforderlich ist, vor Beginn der Bohrung geologische Studien durchzufuehren.

$\mathrm{Zu}$ den durchgefuehrten Verbesserungen zaehlen folgende: Anwendung von Schleifscheiben, wodurch der Ersatz der statischen Druckkraft durch dynamische Vorgaenge erreicht wird; die Anordnung von Wasserstrahl bei Hochdruck neben Bohrwerkzeugen, womit die Schnittleistung erhoeht wird; die Anwendung von Hydrauliktransport bei Stollen von kleinen Durchmessern; die Anwendung von Aminoplaste mit Glasfaser verstaerkt zur Erhoehung der Dichtigkeit und zur Verbesserung der Verkleidung der Tunnel; usw. 